\title{
El reconocimiento a la docencia universitaria: la visión del Estado, del mismo profesor y de la sociedad*
}

\author{
Recognition of University Teaching: The Vision of the State, \\ the Professor and Society
}

Benigno Benavides Martínez**

\begin{abstract}
RESUMEN
El presente estudio aborda el problema del reconocimiento de la docencia universitaria a través de la reflexión teórica acerca de la ética del reconocimiento y de la sociología. La base empírica se obtuvo de datos recolectados mediante encuestas a profesores de universidades públicas y privadas de Monterrey, México. El análisis contrasta las diversas funciones que debe cumplir el profesor universitario, enfocándose en la diferenciación provocada al tener que actuar como investigador y como docente, como si fueran tareas equivalentes. Debido a que a la investigación se le confiriere mayor estatus, la docencia ocupa un lugar secundario, lo cual fundamenta la idea de que ésta carece del reconocimiento social necesario para constituir la identidad profesional del profesor universitario, y, con base en ello, se problematiza su compromiso con las políticas gubernamentales. Los hallazgos muestran que los profesores valoran la función que desempeñan como docentes, integrando su identidad en torno a esta función además de sentirse reconocidos por los demás; pero, por otro lado, se sienten escasamente reconocidos por el Estado. La interpretación sugiere la necesidad de reconocimiento para el desempeño ético de la profesión.
\end{abstract} Palabras clave: docencia universitaria, ética del reconocimiento, ética profesional.

\footnotetext{
* Los resultados que aquí se presentan forman parte de una investigación más amplia acerca del reconocimiento a la docencia universitaria, en la que se consideran más entidades y se extiende el número de la muestra.

** Doctor en Ciencias Sociales por la Universidad Autónoma de Nuevo León, México. Contacto: benigno.benavidesmrt@uanl.edu.mx benignobenavides@gmail.com ORCID: 0000-0002-2515-0377
} 


\begin{abstract}
This study address the problem of recognizing university teaching through theoretical reflection on the ethics of recognition and sociology. Empirically, it is based on data collected from surveys applied to public and private university professors in Monterrey, Mexico. The analysis contrasts the different roles and functions met by the university professor, focusing on the differentiation caused by having to act as a researcher and as a teacher, as if they were equivalent tasks. Because research gives professors a greater status, teaching occupies a secondary position, which supports the idea that it lacks the necessary social recognition to constitute the professional identity of a university professor and, based on this, his or her commitment to government policies is questioned. The findings show that professors value the role they play as teachers, integrating their identity around this function, and they also feel recognized by others; but, on the other hand, they feel little recognition from the State. The interpretation suggests that the ethical performance of the profession is associated with a need for recognition.
\end{abstract}

Key words: universit y teaching, ethics of recognition, professional ethics. 


\section{Introducción}

La docencia es la menos reconocida de las funciones que realiza el profesor universitario; la investigación, en cambio, es ampliamente aceptada como meritoria. Las razones de esta diferencia son diversas: por un lado, la docencia sigue siendo una práctica tradicional, aunque los principios pedagógicos en los que se sustenta sean nuevos y dinámicos. Como docente, el profesor debe trabajar en beneficio de sus estudiantes, ofreciéndoles todos los contenidos de aprendizaje de una forma aprehensible (Alfaro-Rocher \& Pérez Boullosa, 2011), a pesar de que ocasionalmente aquellos se resistan a prestarle atención y lo vean como alguien a quien deben soportar; la docencia es algo que muchos hacen, que otros tantos han hecho y que otros más pueden hacer, mientras que la investigación es siempre nueva, no depende de motivar el interés de otros para captar su atención y esperar un buen aprendizaje, sino que se trata específicamente de someterse por sí mismo a una disciplina de trabajo, a una actividad de búsqueda de interrogantes y de confección de respuestas o soluciones, siguiendo los principios de la investigación científica o humanística. La investigación logra alcanzar productos tangibles y los presenta a la comunidad científica como informes, artículos en revistas de circulación internacional y registro de patentes, los cuales propician el acceso a distinciones de prestigio para los investigadores, a través de eventos como la asistencia a congresos internacionales, la obtención de grados, ofertas de nuevos empleos en universidades de mayor relieve y premios diversos, así como sustanciales incrementos en los ingresos económicos; la investigación, a diferencia de la docencia, es una actividad que no cualquiera puede hacer, solo los científicos con una sólida formación y experiencia la pueden llevar al cabo. Ambas son actividades que se realizan en la universidad y por parte de los mismos profesores, las dos requieren de grandes esfuerzos, pero las recompensas, sean materiales o simbólicas, son muy desproporcionadas, siempre en contra de la docencia.

Docencia e investigación son las dos grandes formas de actividad académica (Clark, 1987) que se realizan en las universidades por los mismos profesores, bajo el supuesto de que ambas, por una supuesta igual naturaleza, se pueden realizar como si fueran parte de un mismo trabajo. Sin duda que la investigación y la docencia son actividades académicas y que la universidad es la institución que se dedica a su 
realización, pero eso no quiere decir que de ello se derive automáticamente un nuevo rol que las integre a ambas en la persona del profesor.

En la actualidad, la expansión de la educación superior y la consecuente masificación de sus actores, tanto estudiantes como profesores, han vuelto a la profesión académica igualmente masiva, sobre todo en el caso de la docencia (Altbach, Reisberg \& Rumbley, 2009). Esta situación se agudiza en las universidades de países no desarrollados y con elevado número de población joven, en donde se considera a la educación como una forma de ascenso social y económico, por lo que ingresar a una universidad se hace, sobre todo, con el fin de estudiar una carrera profesional para ubicarse en el mercado laboral en mejores condiciones que quienes no cuentan con dichos estudios. Las universidades, en este contexto, se han enfocado principalmente a la formación de profesionistas para atender las demandas sociales sobre la educación, postergando para un mejor momento las tareas de investigación. En este sentido, los profesores se conciben predominantemente como docentes. Con la transformación de las universidades en México, a partir de las políticas gubernamentales de la calidad (Asociación Nacional de Universidades e Instituciones de Educación Superior, 1999) éstas se enfocaron hacia el alcance de metas preestablecidas en planes de desarrollo, especialmente confeccionados para mejorar la calidad de la educación superior, incluyendo en este propósito las tareas de la investigación a las cuales se dedicaron los esfuerzos de los profesores, quienes identificaron esta actividad como prestigiosa y redituable.

En el contexto de la búsqueda de calidad, la docencia permaneció estancada en las prácticas tradicionales, aunque revestida de herramientas tecnológicas y autoidentificada con discursos psicopedagógicos de vanguardia (Zabalza, 2004) que pregonaban la centralidad del estudiante en la enseñanza, quien debía acceder al dominio de una amplia gama de competencias y el aprender a aprender como su nueva característica, dentro de una universidad reconocida por su calidad.

Parece evidente que la docencia no es una actividad muy valorada, a pesar de los discursos pedagógicos y de las declaraciones gubernamentales que ubican a la educación como la solución a futuro para prácticamente todos los problemas sociales. Lo que dejan siempre pendiente estos juicios son las estrategias concretas que se habrán de 
seguir y los recursos que se deben invertir para transformar la enseñanza tradicional en otra, acorde con los principios pedagógicos que sustentan su misión organizacional, superando la simple implantación de herramientas informáticas cada vez más sofisticadas, y dejando en segundo plano la transformación de las interacciones de carácter pedagógico que se suscitan en el aula como espacio de cotidianeidad.

El tema central de este estudio es el reconocimiento al profesor universitario en su papel de docente, sin tomar en cuenta su actividad como investigador. Este tema enfatiza la idea de que no es suficiente la simple acción de enseñar del profesor, sino que su buen desempeño requiere de cierta forma de retroalimentación, que le provea información y, sobre todo, que sienta que es apreciado, no solo por su labor, sino sobre todo como persona. Evidentemente que el reconocimiento es un acto que puede tener elementos objetivados, pero se compone, sobre todo, de elementos intersubjetivos. En este sentido, el individuo que recibe el reconocimiento debe otorgarle valoración a ese acto, sintiendo aprecio por lo recibido, lo cual se proyecta recíprocamente a quien hizo el acto de reconocimiento.

El individuo actúa relacionalmente con los demás con base en estos reconocimientos, constituyendo el fundamento de la vida social, puesto que, al hacerlo descansar en lazos subjetivos, además de los objetivos, consolida el sistema de relaciones; de otro modo, la sociedad solo se constituiría por relaciones sin valoración, dejando expuesta la solidez de estas relaciones a la conveniencia de cada una de ellas. Las relaciones sociales tienden a permanecer con cierta persistencia, puesto que cada acción, una vez que se establece, se consolida como algo valorado y, por lo tanto, repetible para futuras interacciones, lo cual convierte al reconocimiento en una especie de fundamento de la vida social (Honneth, 1997).

El esquema básico del reconocimiento sería el que se establece entre individuos que entran en interacción, en la que ambos reconocen al otro para luego ampliar los reconocimientos a instituciones y tradiciones que pueden llegar a convertirse en una base histórica de la sociedad. Especialmente importante en la sociedad actual es el reconocimiento que se brinda el propio individuo por sí mismo, aunque este objeto de reconocimiento no es nuevo, puesto que es fundamento del esquema de reconocimiento social que existe desde el mismo ori- 
gen de la sociedad, es en la actualidad cuando adquiere mayor relieve, debido al lugar preponderante que adquiere el individuo al lado de las instituciones y papeles preconcebidos de la sociedad en estos tiempos. El reconocimiento a sí mismo es también importante, porque es la forma como el individuo establece las expectativas de reconocimiento de los demás; bajo la lógica de que sus acciones son reconocidas por él mismo, es posible que los demás también las reconozcan.

Aunque las relaciones sociales sean establecidas perdurablemente y lleguen a convertirse en una especie de base de acción para la misma sociedad, el reconocimiento puede ser negado en intensidad o en frecuencia, de acuerdo con ciertas situaciones suscitadas entre los actores. El reconocimiento puede ser negado o minimizado debido a que, ciertos actores, pretenden concentrar los reconocimientos hacia sí mismos o hacia algunos individuos en particular, suscitando una especie de disputa por lograr el reconocimiento negado o disminuido. La falta de reconocimiento se presenta, sobre todo, cuando emergen situaciones nuevas que algunos actores desean les sean reconocidas y que antes no se habían hecho manifiestas como algunos rasgos de individualidad.

El reconocimiento por parte de sí mismo y de los demás, dentro del campo laboral de las profesiones, se considera como un elemento básico para mejorar su desempeño y, en el caso de la docencia, como un asunto fundamental en el propósito de elevar la calidad de la educación superior en México, debido al escaso reconocimiento del que ha sido objeto el docente universitario, sobre todo en la época de expansión de la matrícula de la educación superior. Este problema se encuentra relacionado directamente con la identidad del profesor como docente, pues su reconocimiento debe contar con un referente que interactúe con los individuos o instituciones que lo reconocen.

De la identidad se deriva, a su vez, el auto reconocimiento del profesor y la importancia que otorga a su trabajo. Junto a los referentes de sí mismo, se puede identificar otros referentes importantes para los docentes, como el Estado, la principal institución que se involucra con el reconocimiento al docente, debido a su papel en la dirección de la educación, y posteriormente debemos estimar el reconocimiento que el profesor recibe de la sociedad en general, a través del juicio de los otros. 
Bajo la perspectiva descrita se enfoca el presente estudio, ubicando al profesor universitario en cuanto a su identidad como tal, para pasar a estimar el reconocimiento que tiene hacia sí mismo y el que siente que recibe de parte del Estado y de los demás. El trabajo empírico se sustenta en encuestas aplicadas a profesores universitarios que en 2018 se encontraban actuando como docentes de licenciatura, en modalidad presencial en la ciudad de Monterrey, México.

\section{Fundamentos teóricos}

La docencia en las universidades constituye un campo ocupacional de primer orden dentro del mercado laboral, a pesar de las dificultades para ubicarla como una profesión (Sancho-Gil, 2001), debido a la multiplicidad de tareas poco definidas que debe cumplir el profesor, y a la falta de un conocimiento disciplinar específico que confiera unidad a la ocupación. Si bien la profesión de profesor universitario no está completamente definida, se puede considerar cierta homogeneidad ocupacional en cuanto a la función que cumple y las competencias necesarias para su desempeño (Mas-Torelló, 2011), por lo que, en este sentido, se puede hablar más propiamente de un grupo ocupacional. La unidad de grupo queda confirmada con la adscripción colectiva a una misma dirección administrativa por parte de las autoridades de la universidad, lo cual confiere igualmente unidad laboral.

Las profesiones, desde su propio concepto, se fundan en el reconocimiento de la sociedad para hacer efectiva la posibilidad de servir a una clientela demandante de sus servicios (Gorman \& Sandefur, 2011), característica que debe ser compartida por la profesión de profesor universitario. Las profesiones se encuentran permanentemente en una dinámica de formación y transformación, pero en general tienden hacia un proceso que ha sido conceptualizado como "profesionalización", que abarca las ocupaciones de la sociedad en su conjunto, dirigiéndolas hacia su formalización en los diversos ámbitos, hasta ser incluidas en el currículo universitario como carreras para la formación profesional universitaria (Wilensky, 1964). La idea de "profesionalización" establece como una de sus características fundamentales la del reconocimiento social de la profesión, independiente de la rigurosidad científica de los conocimientos o del uso de tecnologías innovadoras. Lo que más interesa a los clientes y a la sociedad es que efectivamente 
el profesional pueda proveer el servicio requerido en el nivel deseado. Si cumple con esta demanda, el profesionista recibirá el reconocimiento necesario para mantenerse en el ejercicio de su profesión.

En esta perspectiva se destaca la funcionalidad de la profesión y, por consecuencia, su necesidad de reconocimiento. Este reconocimiento se puede expresar de varias formas, recorriendo tanto expresiones materiales como simbólicas. En un sentido funcionalista (Davis \& Moore, 1945), se distribuye de forma gradual de acuerdo con la importancia concedida a la función que cumple o a lo que significa aquello que se está reconociendo, de tal modo que los individuos, al igual que las instituciones o medios tecnológicos, se acomodarán en una especie de jerarquía que abarca a toda la sociedad, por lo que ésta se visualiza bajo la forma de estratificación, confiriendo prestigio y poder de manera diferenciada, lo cual pone el escenario necesario para se presente una especie de competencia entre las diversas profesiones para lograr reconocimiento. En el caso de las profesiones, la sociedad las reconoce desde el momento en que alguien, pudiendo ser un cliente, establece una relación con un profesionista para solicitar su servicio y le ofrece un pago, pero a la vez que las reconoce, establece diferencias entre ellas; algunas son de mayor relevancia o urgencia que otras, por lo que deben ser retribuidas a través de incentivos económicos graduales, y se distribuyen rasgos de prestigio discriminadamente. Aunque las profesiones sean una función general, deben ser desempeñadas por un amplio número de individuos con características propias y variables, provocando que el reconocimiento se haga aún más diferenciado, hasta llegar a especificarlo en cada individuo que se desempeña como profesionista.

El reconocimiento que se dispensa a las profesiones también varía atendiendo a circunstancias sociales, como las políticas estatales sobre educación, la prosperidad económica, la expansión de la educación superior, la competencia entre los profesionistas, la demanda de los contratantes, la organización gremial de la profesión y otras. Pero de todas las variantes, en el caso de la profesión docente, exceptuada la del reconocimiento a sí mismo, dos de ellas se extienden con mayor influencia que las otras: por un lado, el papel que asume el Estado como el principal promotor de la educación y como máxima autoridad en este ámbito, dictando políticas, manejando presupuestos, abriendo escuelas y fomentando la participación de los particulares; los profe- 
sores son parte fundamental de las políticas educativas impulsadas por el Estado, por lo que la relación entre ambas entidades resulta de primordial importancia. Por otro, la constitución de gremios hace que muchas veces las individualidades se diluyan, que las relaciones laborales se hagan de manera colectiva, controlando salarios, puestos y promociones, y terminando por burocratizar la actividad profesional. Aunque no se pretenda, la acción del Estado y el sentido gremial hacen que la identidad como docentes o académicos se integre y hasta se fortalezca, adoptando la nueva profesión con base en la ocupación colectiva. En el caso latinoamericano, con la expansión de la educación superior (García-Guadilla, 2002) y la promoción del Estado de este nivel educativo a través de la apertura de universidades públicas, la identidad del profesor se identifica con el sector público y con las políticas gubernamentales, lo mismo que su organización gremial, lo que le permite acceder a los beneficios de los trabajadores de gobierno y a consolidar su carácter gremial, debiendo sujetarse, sin embargo, a la organización casi corporativa predeterminada por el Estado para toda la burocracia.

El sentido gremial desempeña un papel muy importante en el caso de los profesores, sobre todo de quienes trabajan en universidades públicas. El profesor es contratado para ocupar un puesto laboral que ya existe y que tiene prácticamente definidas sus tareas y horarios. Las actividades y reglas y horarios son igualmente cumplidos por un número significativo de individuos, bajo las mismas normas y bajo la misma dirección, asumiendo una fuerte burocratización. Las oportunidades de individualización en este ambiente quedan prácticamente canceladas, al menos al interior de la institución, por lo que la identidad se asume de manera colectiva y las trayectorias laborales siguen las esquematizaciones ya trazadas desde la institución. Lo que se desprende de esta situación, para el propósito de este estudio, es la cobertura colectiva que se hace al conceder el reconocimiento primeramente a toda la profesión y de ahí desprenderlo a cada individuo, lo que significa que cuando excepcionalmente se dirige hacia un individuo es porque éste ha cumplido en un alto nivel las expectativas del conjunto.

La composición gremial del profesorado conduce al acceso colectivo a los mecanismos de ascenso laboral y a las diversas formas de reconocimiento, las cuales son frecuentes en el ámbito institucional de las uni- 
versidades. Estos logros se estiman en el posicionamiento jerárquico y en los logros materiales, pero resultan siempre insuficientes a juicio de los profesores (Aitken \& Tatebe, 2014). El reconocimiento puede ser habitualmente reducido a una política institucional de recompensas, sobre todo materiales, por el cumplimiento destacado de las normas establecidas y el logro de metas fijadas previamente, pretendiendo cumplir con ello por lo menos con una política institucional de reconocimiento. El profesor puede interiorizar estos esquemas, asumiendo el prestigio de esas recompensas como sus propios logros a los cuales accede por sus méritos, abandonando con ello la posibilidad de generar otros esquemas más acordes con su individualidad y profesionalismo.

Sobrepasando el nivel de las recompensas materiales y de las mejoras gremiales, el reconocimiento es un componente fundamental de la vida educativa, pues es difícil pensar siquiera en que la enseñanza o el aprendizaje puedan verificarse si no se reconoce que el profesor está transmitiendo algo valioso, digno de ser aprendido, y que está cumpliendo mucho más que con un simple deber. Por su parte, el profesor reconoce que los estudiantes están tratando de aprender para integrarse a la vida laboral a través de su profesión, y que igualmente realizan su mejor esfuerzo. El reconocimiento, en este sentido, establece vínculos que consolidan a los individuos dentro de las relaciones sociales de manera más sólida de lo que lo hacen las relaciones formales de la escuela.

El reconocimiento, al ser atribuido además de la manera colectiva a profesores específicos en cierto momento de la vida universitaria, supone que se puede presentar la falta de reconocimiento, o presentarse como forma negativa de identificar a los demás individuos que no han sido objeto del reconocimiento, pero que desempeñan roles en las instituciones; de modo que el reconocimiento se convierte en un proceso contradictorio (Huttunen \& Heikkinen, 2006) en el que convergen situaciones favorables o situaciones que lo entorpecen. La vida universitaria propicia ambas situaciones. Aunque formalmente el reconocimiento es siempre positivo, la contradicción se evidencia cuando se otorga a unos profesores, mientras que otros se ven privados de ese reconocimiento, estableciendo, de hecho, una diferenciación entre ambos grupos. Estar inscrito en la clase del profesor reconocido como "el mejor" es sin duda gratificante para todos sus estudiantes, pero es de hecho un reconocimiento negativo para todos los demás profesores y 
así es percibido y valorado por los estudiantes. La formas negativas del reconocimiento se pueden expresar también de manera objetiva como castigos, sanciones, descuentos salariales o advertencias de parte de las autoridades de la institución por faltas contra la normatividad establecida; pero lo que nos interesa sobre todo acerca del reconocimiento negativo es que no tiene versiones formales o establecidas, pero que se manifiesta directamente y en toda ocasión en la vida cotidiana de la institución, con reacciones de apatía, ausentismo estudiantil e indiferencia hacia los docentes.

Como ha sido expuesto hasta este punto, hemos situado al reconocimiento en torno al profesor universitario como uno de los elementos básicos para alcanzar los logros educativos, pero el reconocimiento se extiende a toda la vida social como fundamento para consolidar las relaciones sociales, más allá de la conveniencia económica o de la simple cooperación funcional.

La integración de los individuos o de grupos específicos a la sociedad no es solo un asunto legal de ciudadanía sino un asunto ético que garantiza la solidez de la unidad social. Si la diversidad es uno de los principales elementos que enriquecen a una sociedad, entonces se da por aceptado que se deben integrar grupos e individuos diferentes a los de las élites que hegemonizan la dirección cultural de la sociedad. En este sentido, se puede considerar que el reconocimiento es una lucha entre individuos (Taylor et al., 1994) disputándose un bien intangible, pero atribuible a un gran número de individuos, y que por ello se integra en la identidad profesional del individuo y de una profesión en general. El reconocimiento no se agota al ser atribuido a una cantidad pequeña o grande de individuos, como sucedería en el caso de los bienes materiales o recompensas, que son limitadas y específicas por definición, y, por lo tanto, siempre escasas. El reconocimiento es un bien simbólico compartido por una sociedad como forma de cohesión, necesario para su funcionamiento. Si bien es diferente a las recompensas materiales, comparte con ellas la característica de marcar diferencias y de jerarquizar a la sociedad. Reconocer a otro individuo o profesión equivale a valorarlo socialmente y aceptarlo en la sociedad, sin desgastar sus bienes materiales. Pero la situación contraria del no reconocimiento, a pesar de no repercutir en los bienes materiales, puede negar o por lo menos alejar la aceptación social. 


\section{Metodología}

El desarrollo del presente estudio se orientó hacia el análisis de aquello que los profesores universitarios perciben como reconocimiento a su función docente de parte de la sociedad, del Estado y de sí mismos. Se estimó como pertinente su realización debido al masivo crecimiento de esta ocupación, tanto en instituciones públicas como privadas, desempeñada por profesionistas egresados de educación superior quienes no se insertaron en el campo laboral propio de su profesión o que lo hicieron parcialmente. Desde esta perspectiva, el hecho de que un profesionista formado con ciertas competencias deba recurrir a un campo laboral que requiere competencias muy diferentes, obviamente demanda de cierto reconocimiento tanto para él como para la profesión de la docencia universitaria. Con base en los fundamentos teóricos descritos en el apartado anterior, se realizó el levantamiento de los datos empíricos mediante una encuesta confeccionada para tal propósito.

El cuestionario se integró con cinco apartados referentes al reconocimiento al docente, además de los datos generales de la población estudiada. Las secciones que constituyen el cuestionario se refieren al reconocimiento que los profesores identifican recibir de parte de ellos mismos como docentes, de los directivos de la institución, de parte del Estado y de sus estudiantes. Además, se agregó un apartado con preguntas acerca de los datos generales de los profesores.

Cada uno de las cinco secciones del cuestionario se desglosó en cinco ítems y cada uno de ellos presenta cuatro opciones en una escala que expresa el nivel de reconocimiento que el profesor piensa que recibe. Además de los ítems, se indaga acerca de la importancia que para el profesor tiene la opción seleccionada para los demás ámbitos de su vida, por lo que se agregó otra escala para tratar de averiguar ese nuevo nivel de reconocimiento (Burke et al., 2016). Se trata de una investigación de carácter exploratorio y la muestra se constituyó de 120 profesores.

\section{Resultados}

Los resultados que se presentan en este estudio constituyen el avance de un proyecto que tiene continuación, por lo que, en este caso, se analizan los datos de tres aspectos del reconocimiento que se otorga 
al docente universitario. El primero de ellos se refiere a la importancia que ellos mismos otorgan a su función docente; el segundo al reconocimiento de parte del gobierno y el tercero indaga acerca del reconocimiento de la sociedad en general respecto de su trabajo, iniciando con los datos generales de los profesores. Siguiendo este orden se presentan a continuación los resultados y su análisis.

\subsection{Datos generales}

El total de los profesores que contestaron la encuesta fue de 31, (20 profesores y 11 profesoras,) quienes se desempeñan como docentes en alguna universidad del área de Monterrey, México. En esta actividad tienen una antigüedad que va de los tres hasta los 38 años. La mayor parte de la antigüedad como profesores (90\%) alcanza hasta los 25 años, restando solo tres casos de mayor cantidad de años de servicio. Las profesiones que estudiaron los profesores son predominantemente de Ciencias Sociales y de Ciencias de la Salud, aunque también se encuestó a ingenieros y profesionistas de Ciencias Exactas. La edad va de los 28 a los 73 años, aunque el $80 \%$ se ubica hasta los 50, más allá de esta edad solo se registraron seis casos. El grado académico de los profesores es el de Maestría, sumando 24 (77,4\%), un profesor con Doctorado y los restantes con Licenciatura.

Los datos anteriores nos muestran que la docencia en la universidad es una actividad consolidada, ejercida por profesionistas de variados campos del conocimiento, quienes se han formado para su desempeño mediante el estudio de posgrados, con trayectorias laborales firmes y quienes presumiblemente adoptan la docencia como una de las actividades fundamentales para su actividad profesional. La docencia universitaria se desempeña por mujeres y hombres durante lapsos prolongados, con edades que se extienden desde la juventud hasta la madurez, otorgando las bases propias de su identidad profesional.

\subsection{Reconocimiento de la importancia de la docencia}

El primer aspecto por considerar en la problemática del reconocimiento social es el relativo a la importancia en general que el docente le reconoce a su propio trabajo de enseñanza, debido a que debe ser él mismo quien primordialmente establezca la valoración de su función. Aparte de la docencia, el profesor universitario debe realizar 
actividades de investigación, prácticas, tutorías, desempeño profesional y administración, entre otras igualmente importantes, y a las que igualmente concede reconocimiento, por lo que la docencia es solo una función entre otras varias. Aunque en muchos casos y en universidades privadas pequeñas apenas se realice la docencia como la única función del profesor, se considera que su perfil de desempeño debe integrar por lo menos docencia e investigación, para poder dar a la docencia de educación superior una identidad diferente a la de otros niveles educativos.

De acuerdo con la metodología utilizada, se ofreció a los encuestados un conjunto de cuatro juicios en escala en cada ítem acerca del reconocimiento, de los cuales debían seleccionar solo uno. El juicio más seleccionado en este aspecto fue el ubicado en la tercera posición, recibiendo el mayor número de frecuencias (18), y se enuncia como: "mi trabajo como docente es muy importante". Pero el juicio que expresaba el mayor aprecio por la docencia se enunciaba como: "mi trabajo como docente es imprescindible y muy estimado por todos" el cual fue seleccionado solo en diez ocasiones. Con base en lo anterior, se puede inferir que el trabajo docente resulta importante para los profesores, aunque no totalmente imprescindible o muy estimado por la gran mayoría, por lo que se considera que los profesores estiman un moderado reconocimiento por su trabajo.

Si la importancia concedida al trabajo docente es reconocida, entonces es de suponer que el profesor valore igualmente su asistencia diaria a clases para que pueda verificarse su actividad docente. Se esperaría, dados los resultados del ítem anterior, que la asistencia fuera igualmente valorada, pero disminuyó parcialmente al contabilizar 10 frecuencias el juicio de mayor importancia enunciado como: "por ningún motivo falto a clase, todo el tiempo es importante para el curso" mientras que el siguiente juicio "nunca falto a clase, de no ser por algún asunto importante" fue seleccionado en 12 ocasiones. Los otros dos juicios restantes, que expresan situaciones de escasa valoración de la asistencia, también fueron seleccionados, destacando el enunciado como "no importa que yo falte a clase, de todos modos, alcanzo a impartir todos los temas", que registró cuatro frecuencias.

Los profesores, por lo tanto, no sienten el mismo aprecio por su asistencia que la que estiman por su docencia, aunque resulte contras- 
tante, llegando al extremo de no aceptar la importancia de su asistencia y sí resaltar la de la docencia. Las razones de lo anterior pueden ser variadas, destacando, entre ellas, las múltiples actividades que debe realizar el profesor como parte de sus deberes académicos o administrativos, pero de ninguna manera justifican la inasistencia.

La docencia está sujeta a tiempos delimitados por horarios establecidos en los esquemas curriculares, por lo que toda cantidad de tiempo dedicado a la docencia tiene importancia para el logro de los propósitos del curso. Si se pierde, difícilmente se puede recuperar y de ningún modo se puede desperdiciar. Muy ligado al reconocimiento a su asistencia se encuentra el aspecto del aprovechamiento del tiempo, lo cual es un indicativo de la planeación didáctica y el apego al trabajo. Por el contrario, asistir a clase, sin aprovechar el tiempo, se convierte en una simulación. La mayoría de los profesores (20) seleccionaron que "siempre aprovecho el tiempo de mi clase en la enseñanza", sin embargo, ocho profesores seleccionaron un juicio diferente "trato de aprovechar el tiempo de mi clase, pero siempre hay interrupciones", y tres profesores señalaron "debo cubrir el horario de cada curso, aunque no trabaje mucho".

Como se desprende de los datos anteriores, los profesores tampoco tienen el mismo aprecio por el tiempo de su docencia que el que le conceden a la docencia misma. El tiempo de aula del profesor contiene múltiples actividades y está sujeta a interrupciones, en ocasiones envueltas en situaciones propias de la docencia, provocadas por los mismos estudiantes, por los profesores o los administradores. Más compleja sería la falta de valoración del tiempo, aunque relativa, si se debiera a la falta de competencia del profesor para diseñar y poner en acción estrategias de enseñanza que motiven a sus estudiantes, por lo que resulta llamativo que solo cuatro profesores seleccionaran: "no admito ningún tipo de interrupción o distracción en el tiempo de mi clase", ya que era de suponer una más alta valoración por el tiempo efectivo de aula dedicado a la enseñanza.

El contenido de los cursos impartidos en clase tiende, de manera directa o indirecta, a que los estudiantes comprendan que esos contenidos son para su aplicación en situaciones reales de la práctica profesional, aunque el plan de estudios distinga contenidos teóricos, prácticos, talleres, seminarios y otros de carácter filosófico, ético, estético, 
etc., y que muchas veces la gran mayoría de los cursos se conviertan en lecciones orales, independientemente del carácter con el que hayan sido confeccionados. Las situaciones escolares no son las mismas que las que se presentan en la vida profesional, por lo que toda supuesta enseñanza práctica o que tienda a la práctica está situada a cierta distancia de realmente serlo. La aplicación de los contenidos se relaciona con la valoración del trabajo del profesor, haciendo que, mientras mayores posibilidades de aplicación tengan los contenidos que transmite el profesor, pueda ser mayormente valorada su docencia. La mayoría de los profesores (19) se identificaron con el juicio: "acostumbro a explicar a mis estudiantes que lo que enseño se debe aplicar, a pesar de las dificultades", mientras que siete profesores asentaron que "yo mismo y mi trabajo somos ejemplo de que lo que enseño se puede aplicar", evidenciando con ello el aprecio que se tiene por los conocimientos prácticos, aunque en la docencia solo se practiquen técnicas tradicionales de enseñanza.

En atención al reconocimiento de la docencia, el profesor va integrando su identidad profesional y se reconoce a sí mismo bajo el título de su ocupación. En la encuesta, ocho profesores seleccionaron el juicio: "si es necesario, menciono que soy profesor, aunque acompañado de mi título profesional", solo uno "nunca digo que soy profesor, prefiero mi título profesional", y la mayoría (17) optó por la opción "estoy orgulloso de ser profesor".

Las opciones seleccionadas tienden a reconocer la importancia del trabajo orientado hacia la práctica de la profesión y de sí mismos como profesores, aunque no le reconocen la misma importancia a su asistencia a clases, ni al aprovechamiento del tiempo de clase, ni a identificarse como "profesores" antes que en relación con su título profesional.

\subsection{Reconocimiento del papel del gobierno}

El crecimiento de las universidades públicas ha sido impulsado por el gobierno mexicano desde la década de 1970, para responder a la demanda por este nivel de formación profesional; incluso la educación, en este caso, puede ser considerada como una extensión de las funciones de gobierno, generando una afinidad entre las políticas gubernamentales y las orientaciones administrativas de la universidad, dando por establecido un vínculo entre ambas entidades. El profesor 
universitario, consecuentemente, debe percibir el reconocimiento por parte del gobierno hacia el desempeño de sus funciones, debido a la importancia de la formación de nuevos profesionistas a través de la docencia universitaria, lo cual se considera valioso para el desarrollo de la sociedad.

Con el propósito de averiguar el nivel de reconocimiento que los profesores sienten que reciben de parte del gobierno, se presentaron varios ítems en forma de escala. En el primero de ellos se pregunta directamente acerca de este tema. La mayoría de las opciones seleccionadas se agrupan en torno a la propuesta de que el gobierno no reconoce el trabajo de los profesores o solo lo hace en ocasiones como el 15 de mayo (Día del Maestro). Solo un profesor, de todos los encuestados, optó por aceptar que "el gobierno siempre ha reconocido el trabajo de los docentes".

El gobierno realiza su acción política en educación y, para alcanzar sus objetivos, requiere del compromiso y de la participación de todos los profesores, puesto que son ellos los que habrán de concretar las acciones delineadas desde la política estatal en actividades en el aula. "El profesor a veces es tomado en cuenta, pero solo para asuntos sin importancia" es el juicio mayormente seleccionado (16) y 10 profesores seleccionaron "el gobierno es la máxima autoridad en la educación"; por otro lado, solo un profesor asumió que "el gobierno consulta a los profesores antes de tomar decisiones". Los resultados indican que los profesores no son tomados en cuenta en las decisiones de gobierno, por lo que no se puede asumir que se consideren participantes activos en la política educativa, ni sientan reconocimiento significativo de parte del gobierno.

El reconocimiento del gobierno a los profesores se puede extender también a la institución universitaria, lo cual se destaca en un ítem dedicado a este propósito. El planteamiento se hizo partiendo de la idea de que la universidad, al depender del gobierno, cumple con funciones políticas y sociales en favor de éste. De los juicios presentados, solo seis profesores se identificaron con la idea de que "sin la intervención del gobierno, las universidades no podrían funcionar" y ocho se unieron a la idea de que "el gobierno apoya a las universidades para que cumplan con su función". Los otros juicios destacan el papel político de las universidades, como el que se enuncia diciendo: "lo único que le inte- 
resa al gobierno es que las universidades estén en paz, aunque no se enseñe", el cual fue seleccionado en ocho ocasiones y, finalmente, "el gobierno vigila que las universidades efectivamente cumplan con su función", que fue seleccionado con nueve frecuencias.

En un ítem parecido al anterior, los juicios que se ofrecían se refieren a la función de la universidad respecto del Estado, a partir de admitir la autoridad del gobierno en la universidad. El juicio que mayor número de frecuencias registró (17) fue "el gobierno a veces tiene que recurrir a las universidades para cumplir con sus fines". Los juicios que expresan el respeto del Estado por la universidad, en conjunto recibieron 10 frecuencias y, de ellas, el que mayormente reconoce a las universidades fue el de menor frecuencia, mientras que cuatro profesores se identificaron con un juicio contundente: "el gobierno utiliza las universidades para fines políticos".

El último ítem de esta sección indaga acerca del sentido que tiene para el gobierno la admisión de grandes cantidades de población juvenil para recibir los beneficios de la educación superior, a pesar de no existir garantía alguna para que los futuros profesionistas puedan integrarse al mercado laboral. Los resultados muestran que se mantiene la idea de que la educación es la mejor forma de progresar y que, por ello, el gobierno la fomenta, aunque un importante número de profesores (9) seleccionaron la idea de que "el gobierno fomenta la educación solo para que los jóvenes se mantengan ocupados".

\subsection{Reconocimiento de parte de los otros}

Los reconocimientos al trabajo docente provenientes del mismo profesor y del gobierno, como principal actor de la política educativa, son de relieve para el profesor, pero el reconocimiento de los demás es fundamental, pues la profesión solo adquiere sentido si un público generalizado considera sus servicios con la suficiente necesidad como para contratar a un profesional, teniendo que pagar por recibir ese servicio. En el caso de la educación y especialmente hablando de la universidad pública, el supuesto cliente puede ser cualquier persona que reúna los requisitos académicos de ingreso, pero no tiene que pagar los servicios académicos, por lo que la exigencia de cumplimiento de calidad en el servicio no se puede contrastar con pagos diferenciados de acuerdo con el nivel de calidad. El reconocimiento de los otros a la docencia 
no se puede hacer como cliente, pero sí como usuario o beneficiario directo, o a través de familiares, hijos, parientes o conocidos. En el caso de las universidades privadas, el reconocimiento es aún más complicado debido a las escasas prestaciones laborales que ofrecen (comparadas con las públicas), lo que hace que los profesionistas permanezcan poco tiempo trabajando en ellas.

El primer aspecto que se analiza en este apartado es el concerniente a la relación del profesionista como docente, comparado con el profesionista trabajando en tareas específicas de su profesión. Inicialmente se presenta la opción de "me han dicho que, si dejo de ser profesor y me dedico a mi profesión, ganaría mucho más", el cual fue seleccionado por seis profesores, en tanto que el que expresa: "como profesor soy reconocido igual que si trabajara como profesionista" fue el más seleccionado (16). Los juicios que resaltan la profesión docente como más reconocida que otras prácticas profesionales recibieron, en conjunto, menos frecuencias; el juicio que fue menos seleccionado es el enunciado: "realmente pienso que ser profesor es mejor que ser profesionista".

Continuando con el reconocimiento de la docencia en relación con la práctica profesional, se volvió a plantear en un nuevo ítem con los siguientes juicios: "mi trabajo docente es considerado solo como último recurso de empleo"; "mi trabajo docente es mal pagado, pero más seguro que dedicarme a mi profesión"; "mi trabajo docente está bien pagado y soy reconocido como profesionista", y "mi trabajo docente es el más reconocido por formar nuevas generaciones". Los juicios que registraron mayor número de frecuencias fueron los dos últimos, $10 \mathrm{y}$ 11 respectivamente, mientras que los primeros juicios recibieron seis y cuatro selecciones. En general, se puede verificar que la docencia presenta ciertas ventajas laborales y reconocimientos, a pesar de no recibir grandes retribuciones salariales.

La docencia, sobre todo si es el único empleo que desempeña el profesionista, toma cierta distancia de la práctica profesional, debido a que el profesor se especializa en ciertos cursos formales, por lo que pasa la mayor parte del tiempo en las aulas, mientras que la práctica profesional transcurre por otra dinámica. El profesor no necesariamente es la máxima autoridad en los conocimientos de su profesión, aunque, por las características de su trabajo, se encuentra inmerso en 
los adelantos de su disciplina. De esta situación se obtiene entonces que el docente es un experto con poco acceso a la práctica. El reconocimiento de los demás hacia el docente proviene de su conocimiento disciplinar, por lo que se debe indagar acerca de esta característica, para lo cual se plantearon varios juicios que se analizan enseguida.

Los datos muestran una clara tendencia (19 frecuencias) a reconocer que los conocimientos del profesor son el elemento básico para ser considerado apto para la práctica profesional. Los juicios que no reconocen la capacidad del profesor para desempeñarse en la práctica resultaron con muy bajas frecuencias (cuatro en total). Otro ítem selecciona igualmente al conocimiento como la garantía para el trabajo docente. La problemática que surge de la preponderancia del conocimiento para incidir tanto en la práctica profesional como en la docencia consiste en que éste es solo un componente de ambos. En la práctica profesional intervienen muchos elementos de carácter laboral, económico, innovaciones en la organización del trabajo, nuevos materiales, competencia entre oferentes, altibajos en el mercado y demás situaciones, las cuales hacen que el papel del conocimiento de un profesionista individual quede circunscrito al conjunto de los componentes. En el caso de la docencia ocurre algo parecido, agravado por la masificación del personal docente en las universidades, los salarios estandarizados y la convivencia diaria, los cuales repercuten a su vez en la consolidación del espíritu gremial y la formación de la identidad.

Los datos tienden a identificar el reconocimiento a la docencia a través de la capacidad del profesor de asimilar conocimientos propios de su campo con 12 frecuencias, pero, casi con el mismo número, los profesores seleccionaron el juicio: "mi trabajo como docente solo tiene sentido si los estudiantes aprenden", mostrando un sentido de servicio más allá del simple cumplimiento del deber.

\section{Discusión}

El planteamiento inicial que se ha hecho en este estudio establece la premisa de la falta de reconocimiento a la función docente que realizan los profesores universitarios, razón por la cual no se integran cabalmente bajo esa identidad y, sobre todo, no se pueden alcanzar las metas institucionales de calidad educativa emprendidas por el Estado. $\mathrm{El}$ escaso reconocimiento parece sustentarse en la naturaleza misma 
de la docencia, por ser una actividad desempeñada masivamente en la sociedad, en el medio de las escuelas de todos los niveles, por una variedad de agentes, quienes no han sido integrados en el mercado laboral de su profesión de estudio universitario.

En la institución universitaria la función docente se opaca frente a la investigación, aunque subsiste como fundamento de la identidad del profesor, por lo que evidentemente es valorada por los profesores, lo mismo que la orientación a la práctica de los conocimientos profesionales, resultando contrastante que no valoren en la misma medida su asistencia diaria a los cursos, ni el aprovechamiento del tiempo de docencia.

La estrecha relación de la educación con el Estado se estableció desde la fundación de las universidades públicas, por lo que es de esperar que los profesores reciban un amplio reconocimiento por parte del éste; sin embargo, ellos no perciben que el Estado los reconozca. Lo anterior podría ser el efecto de varios factores, como los bajos salarios, la masificación, la gremialización y la verticalidad de las políticas sobre las instituciones universitarias.

Lo más problemático de este asunto se presenta en la ineficacia de las políticas educativas, al establecerse en desconexión con las acciones docentes, anulando de entrada el alcance de los logros establecidos en los proyectos gubernamentales, pues los actores directos de esos proyectos no se sienten reconocidos y, por lo tanto, no se espera que se comprometan con las políticas estatales. La relación de los profesores con el Estado se escapa de la funcionalidad y se alinea con percepciones que presentan a la universidad, más que como una institución de docencia e investigación, como una manipulación de las políticas de gobierno.

La docencia universitaria es valorada como una ocupación por las prestaciones y la seguridad laboral, en contraste con la práctica profesional que se ubica en un campo abierto a los altibajos de la contratación, de las fluctuaciones del mercado laboral y de la economía en general. Si bien el docente no es un experto en las situaciones de la práctica profesional, sí se le puede considerar como especialista en el ámbito de su actividad académica, específicamente en el dominio de los conocimientos de los cursos que imparte, por lo que se afirma a 
este carácter al compararse con los profesionistas que se desempeñan en la práctica cotidiana de la profesión, lo cual le confiere el rasgo de identidad más definida.

La más aguda de las disyuntivas del reconocimiento a la docencia por parte de los otros, se presenta en dos posibilidades: la de ser reconocido como docente o la de ser reconocido como profesionista. Ambas opciones tienen problemas, pero al tratar de diferenciarlas con rasgos definitorios, éstos se presentarían, en el caso de la docencia, como el conocimiento, mientras que para el profesionista sería el de la práctica, por lo que el docente percibe que los demás lo valoran por su conocimiento. Queda pendiente la problemática referente a la práctica de la docencia como un dominio profesional o, simplemente, la continuidad de las formas tradicionales de enseñanza como transmisión mecánica de conocimientos. Pendiente queda también analizar el problema, aún más amplio, de la docencia como formación de los estudiantes que aspiran a ser profesionistas y del compromiso ético que esto implica.

\section{Referencias}

Aitken, G. \& Tatebe, J. (2014). Recognition of Teaching Excellence Discussion paper. Auckland: University of Auckland.

Alfaro-Rocher, I. J. \& Pérez Boullosa, A. (2011). Un Estudio acerca de la dedicación docente del profesorado universitario en el marco del Espacio Europeo de Educación Superior. Revista Electrónica Interuniversitaria de Formación del Profesorado, 41-56.

Altbach, P. G., Reisberg, L. \& Rumbley, L. E. (2009). Trends in Global Higher Education: Tracking and Academic Revolution. A Report prepared for the UNESCO 2009 World Conference in Higher Education. Paris: UNESCO.

Asociación Nacional de Universidaddes e Instituciones de Educación Superior. (1999). La Educación Superior en el Siglo XXI. Líneas Estratégicas de Desarrollo. México DF: ANUIES.

Burke, P. J. (2016). Capability, Belonging and Equity in Higher Education: Developing Inclusive Approaches. New Castle, Australia: The University of New Castle.

Clark, B. R. (1987). The Academic Life. Small Worlds, Different Worlds. A Carnegie Foundation Special Report. Princenton New Jersey: The Carnegie Foundation for the Advancement of teaching. 
Davis, K. \& Moore, W. (1945). Some Principles of Stratification. American Sociological Review, 242-249.

García-Guadilla, C. (2002). Tensiones y Transiciones. Educación Superior Latinoamericana en los albores del tercer milenio. Caracas: Nueva Sociedad.

Gorman, E. H. \& Sandefur, R. (2011). “Golden Age,” Quiescence, and Revival: How the Sociology of Professions Became the Study of Knowledge-Based Work. Work and Occupations, 275-302.

Honneth, A. (1997). La lucha por el reconocimiento. Por una gramática moral de los conflictos sociales . Barcelona: Crítica-Grijalbo Mondadori.

Huttunen, R. \& Heikkinen, H. (2006). Teaching and the dialectic of recognition. Pedagogy, Culture and Society, 163-174.

Mas-Torelló, Ó. (2011). El Profesor Universitario: sus Competencias y Formación. Profesorado: Revista de Currículum y Formación del Profesorado, 195-211.

Sancho-Gil, J. M. (2001). Docencia e Investigación en la Universidad: una Profesión, dos Mundos. Educar, 41-60.

Taylor, Ch. y otros. (1994). Multiculturalism. Examining the politics of recognition. Princenton, New Jersey: Princenton University Press.

Wilensky, H. (1964). The professionalization of everyone? The American Journal of Sociology, 137-158.

Zabalza, M. A. (2004). Innovación en la Enseñanza Universitaria. Contextos Educativos, 113-136. 